PROCEEDINGS OF THE

AMERICAN MATHEMATICAL SOCIETY

Volume 139, Number 11, November 2011, Pages 3789-3794

S 0002-9939(2011)10770-6

Article electronically published on July 12, 2011

\title{
PERIODS OF EISENSTEIN SERIES AND SOME APPLICATIONS
}

\author{
ZACHARY A. KENT
}

(Communicated by Ken Ono)

\begin{abstract}
Bringmann, Guerzhoy, Ono, and the author developed an EichlerShimura theory for weakly holomorphic modular forms by studying associated period polynomials. Here we include the Eisenstein series in this theory.
\end{abstract}

Classically, the periods of a weight $k$ ( $k \geq 4$ will always denote an even integer) cusp form $f=\sum_{l \geq 1} a_{f}(l) q^{l}\left(q:=e^{2 \pi i \tau}\right.$ with $\tau=x+i y \in \mathbb{C}$ with $\left.y>0\right)$ on $\Gamma:=S L_{2}(\mathbb{Z})$ are given by

$$
r_{n}(f):=\int_{0}^{i \infty} f(\tau) \tau^{n} d \tau \quad \text { for integers } n \text { such that } \quad 0 \leq n \leq k-2 .
$$

These periods appear in the coefficients of the period polynomial of $f$ defined by

$$
r(f ; z):=\int_{0}^{i \infty} f(\tau)(z-\tau)^{k-2} d \tau=\sum_{n=0}^{k-2}(-1)^{n}\left(\begin{array}{c}
k-2 \\
n
\end{array}\right) r_{n}(f) z^{k-2-n} .
$$

More generally, we will consider $f=\sum_{l \gg-\infty} a_{f}(l) q^{l} \in M_{k}^{!}$, where $M_{k}^{!}$denotes the space of weakly holomorphic modular forms of weight $k$ (these are meromorphic modular forms whose poles, if any, are supported at cusps). In this case, the integrals in (1) and (2) may be divergent. In [1], Bringmann, Guerzhoy, Ono, and the author found a way to avoid this obstruction by considering the formal Eichler integral of $f$ given by

$$
\mathcal{E}_{f}(z):=\sum_{\substack{l \gg-\infty \\ l \neq 0}} \frac{a_{f}(l)}{l^{k-1}} q^{l} .
$$

The period function of $f$ as defined in [1] is

$$
r(f ; z):=c_{k}\left(\mathcal{E}_{f}(z)-z^{k-2} \mathcal{E}_{f}(-1 / z)\right),
$$

where $c_{k}:=-\frac{\Gamma(k-1)}{(2 \pi i)^{k-1}}$. For a cusp form $f$, this agrees with (2). Therefore, in analogy with (2), we define $r_{n}(f)$, the periods of $f \in M_{k}^{!}$, by

$$
r(f ; z)=\frac{a_{f}(0)}{k-1}\left(z^{k-1}+\frac{1}{z}\right)+\sum_{n=0}^{k-2}(-1)^{n}\left(\begin{array}{c}
k-2 \\
n
\end{array}\right) r_{n}(f) z^{k-2-n} .
$$

Received by the editors August 17, 2010.

2010 Mathematics Subject Classification. Primary 11F67, 11 F03.

The author would like to thank Pavel Guerzhoy, Ken Ono, Don Zagier, and the referee for several suggestions that improved this paper.

(C)2011 American Mathematical Society Reverts to public domain 28 years from publication 
The space $M_{k}^{!}$decomposes into a direct sum of $S_{k}^{!}$(the subspace of $M_{k}^{!}$whose forms have vanishing constant term) and the space generated by the weight $k$ Eisenstein series,

$$
G_{k}(\tau):=-\frac{B_{k}}{2 k}+\sum_{n \geq 1} \sigma_{k-1}(n) q^{n}, \quad \text { where } \quad \sigma_{k-1}(n)=\sum_{d \mid n} d^{k-1},
$$

and $B_{k}$ is the $k$ th Bernoulli number. It was noticed, in [1], that by restricting to forms in $S_{k}^{!}$, the period functions in (4) are actually polynomials. Here, we will study period functions, so it suffices to consider $r\left(G_{k}, z\right)$ and the "extra periods" in (4) that depend on $a_{G_{k}}(0)$.

With respect to previous work relating periods to $L$-values [1, 5, 6, 7, we would like to interpret the "extra periods" in (4) in terms of the $L$-series for $G_{k}$. We do this by following Zagier in [7. Define the $L$-series for $G_{k}$ by

$$
L\left(G_{k}, s\right)=\sum_{n \geq 1} \sigma_{k-1}(n) n^{-s}=\zeta(s) \zeta(s+1-k),
$$

where $\zeta(s)$ is Riemann's zeta function. Then $L\left(G_{k}, s\right)$ has a meromorphic continuation to the complex plane and satisfies the functional equation

$$
(2 \pi)^{-s} \Gamma(s) L\left(G_{k}, s\right)=i^{k}(2 \pi)^{s-k} \Gamma(k-s) L\left(G_{k}, k-s\right) .
$$

Furthermore, $L\left(G_{k}, s\right)$ has a pole only at the point $s=k$, which is simple, with residue $a_{G_{k}}(0)(2 \pi i)^{k} / \Gamma(k)$. Interpreting the binomial coefficient $\left(\begin{array}{c}k-2 \\ n\end{array}\right)$ as the quotient $\frac{\Gamma(k-1)}{\Gamma(n+1) \Gamma(k-1-n)}$, we see that this quotient has a simple zero at each integer $n$ such that $n \notin\{0,1, \ldots, k-2\}$, and the first derivative at the point $n=k-1$ is $-1 /(k-1)$. This motivates the definition of the $n$th normalized period of $G_{k}$ to be

$$
\widehat{r}_{n}\left(G_{k}\right):=\Gamma(k-1) \cdot \frac{i^{n+1}}{(2 \pi)^{n+1}} \cdot \lim _{s \rightarrow n} \frac{L\left(G_{k}, s+1\right)}{\Gamma(k-1-s)} .
$$

Combining the above discussion with identity (3) reveals that

$$
\begin{aligned}
r\left(G_{k} ; z\right) & =c_{k}\left(\mathcal{E}_{G_{k}}(z)-z^{k-2} \mathcal{E}_{G_{k}}(-1 / z)\right)=\int_{0}^{i \infty}\left(G_{k}(\tau)+\frac{B_{k}}{2 k}\right)(z-\tau)^{k-2} d \tau \\
& =\sum_{n \in \mathbb{Z}} \Gamma(k-1) \cdot \frac{i^{1-n}}{(2 \pi)^{n+1}} \cdot \lim _{s \rightarrow n} \frac{L\left(G_{k}, s+1\right)}{\Gamma(k-1-s)}=\sum_{n \in \mathbb{Z}}(-1)^{n} \widehat{r}_{n}\left(G_{k}\right) z^{k-2-n} .
\end{aligned}
$$

In general, for a weakly holomorphic modular form $f \in M_{k}^{!}$, we combine (4) and (5) to define $\widehat{r}_{n}(f)$ (for $n \in \mathbb{Z}$ ), the normalized periods of $f$, by

$$
r(f ; z)=\sum_{n \in \mathbb{Z}}(-1)^{n} \widehat{r}_{n}(f) z^{k-2-n} .
$$

Combining (4), (5), and (6), we find that the normalized periods of $f$ are given by

$$
\widehat{r}_{n}(f):= \begin{cases}\left(\begin{array}{c}
k-2 \\
n
\end{array}\right) r_{n}(f) & \text { for } 0 \leq n \leq k-2 \\
-a_{f}(0) /(k-1) & \text { for } n=-1, k-1, \text { and } \\
0 & \text { for } n<-1 \text { or } n>k-1 .\end{cases}
$$

It is natural to consider an Eichler-Shimura isomorphism in this context extending the work of [1, 5, 7]. In this direction, let $\mathbf{V}_{k}$ be the space of polynomials in $z$ of 
degree at most $k-2$, and define the larger space $\widehat{\mathbf{V}}_{k}:=z^{-1} \cdot \mathbf{V}_{k+2}$. There is an action of $\gamma=\left(\begin{array}{ll}a & b \\ c & d\end{array}\right) \in \Gamma$ on any function $g$ given by $\left(\left.g\right|_{w} \gamma\right)(z):=(c z+d)^{-w} g\left(\frac{a z+b}{c z+d}\right)$. Furthermore, we can extend this action linearly to the group ring $\mathbb{Z}[\Gamma]$, allowing us to define the period function and period polynomial spaces by

$$
\widehat{\mathbf{W}}_{k}:=\left\{P \in \widehat{\mathbf{V}}_{k}:\left.P\right|_{2-k}(1+S)=\left.P\right|_{2-k}\left(1+U+U^{2}\right)=0\right\},
$$

and $\mathbf{W}_{k}:=\mathbf{V}_{k} \cap \widehat{\mathbf{W}}_{k}$, respectively, where $S:=\left(\begin{array}{cc}0 & -1 \\ 1 & 0\end{array}\right)$ and $U:=\left(\begin{array}{cc}1 & -1 \\ 1 & 0\end{array}\right)$ are the standard generators for $\Gamma$ of orders 2 and 3. The authors in [1] showed that $r(f ; z) \in \mathbf{W}_{k}$ for any $f \in S_{k}^{!}$. Zagier showed in [7] that $r\left(G_{k} ; z\right) \in \widehat{\mathbf{W}}_{k} \backslash \mathbf{W}$. Combining these results, it follows that $r(f ; z) \in \widehat{\mathbf{W}}_{k}$ for every $f \in M_{k}^{!}$.

Remark 1. Explicitly, the relations defining $\widehat{\mathbf{W}}_{k}$ are

$$
\sum_{n=-1}^{k-1} a_{n} X^{n} \in \widehat{\mathbf{W}}_{k} \Leftrightarrow a_{n}=(-1)^{n-1} a_{k-2-n}, \quad \text { and } a_{-1}+(-1)^{n} \sum_{m=-1}^{k-1} c_{m n} a_{m}=0 \text {, }
$$

where

$$
-1 \leq n \leq k-1 \quad \text { and } \quad c_{m n}= \begin{cases}\left(\begin{array}{l}
m \\
n
\end{array}\right), & \text { if } m \geq n, \\
\left(\begin{array}{c}
k-2-m \\
k-2-n
\end{array}\right), & \text { if } m \leq n .\end{cases}
$$

The space $\widehat{\mathbf{V}}_{k}$ splits as a direct sum $\widehat{\mathbf{V}}_{k}=\widehat{\mathbf{V}}_{k}^{+} \oplus \widehat{\mathbf{V}}_{k}^{-}$of even and odd functions. This splitting induces further splittings of the subspaces $\widehat{\mathbf{W}}_{k}=\widehat{\mathbf{W}}_{k}^{+} \oplus \widehat{\mathbf{W}}_{k}^{-}$and $\mathbf{W}_{k}=\mathbf{W}_{k}^{+} \oplus \mathbf{W}_{k}^{-}$. Now for any $f \in M_{k}^{!}$, we may write $r(f ; z)=r^{+}(f ; z)+r^{-}(f ; z)$ with $r^{ \pm}(f ; z) \in \widehat{\mathbf{W}}_{k}^{ \pm}$. Denote the space of weight $k$ holomorphic modular (resp. cusp) forms by $M_{k}$ (resp. $S_{k}$ ). Then the classical Eichler-Shimura isomorphism (see [5]) says that we have isomorphisms $r^{ \pm}: S_{k} \rightarrow \mathbf{W}_{k}^{ \pm}$. In [7], Zagier extended this to show that the maps $r^{ \pm}: M_{k} \rightarrow \widehat{\mathbf{W}}_{k}^{ \pm}$are isomorphisms. Here, we extend things further to produce an Eichler-Shimura isomorphism theorem for $M_{k}^{!}$making use of the differential operator $D:=\frac{1}{2 \pi i} \frac{d}{d z}$, which satisfies, by a well-known identity of Bol (see Theorem 1.2 of [2]),

$$
D^{k-1}: M_{2-k}^{!} \longrightarrow S_{k}^{!} .
$$

Theorem 2. Let $r: M_{k}^{!} \rightarrow \widehat{\mathbf{W}}_{k}$ be the period function map. Then we have an exact sequence

$$
0 \longrightarrow D^{k-1}\left(S_{2-k}^{!}\right) \longrightarrow M_{k}^{!} \stackrel{r}{\longrightarrow} \widehat{\mathbf{W}}_{k} \longrightarrow 0 .
$$

Proof. The surjectivity of the restricted map $r: S_{k}^{!} \rightarrow \mathbf{W}_{k}$ was established in [1]. This together with the observation in $\left[7\right.$ that $r\left(G_{k} ; z\right) \in \widehat{\mathbf{W}}_{k} \backslash \mathbf{W}_{k}$ gives us exactness of the sequence at $\widehat{\mathbf{W}}_{k}$. Exactness at $D^{k-1}\left(S_{2-k}^{!}\right)$follows from Bol's identity (8).

Now, let $f \in M_{k}^{!}$. A simple calculation shows that $r(f ; z)=c_{k} \alpha\left(z^{k-2}-1\right)$ for some $\alpha \in \mathbb{C}$ if and only if $\mathcal{E}_{f}+\alpha \in M_{2-k}^{!}$, i.e. $f \in D^{k-1}\left(M_{2-k}^{!}\right)$. Thus the kernel of the period function map is $D^{k-1}\left(S_{2-k}^{!}\right)$, which proves exactness at $M_{k}^{!}$.

Combining Theorem 2 with the isomorphisms $r^{ \pm}: M_{k} \rightarrow \widehat{\mathbf{W}}_{k}^{ \pm}$in [7, we have

$$
M_{k}^{!} / D^{k-1}\left(S_{2-k}^{!}\right) \cong \widehat{\mathbf{W}}_{k} \cong M_{k} \oplus M_{k} .
$$

In contrast, we may start with the following isomorphism considered in [1] and [3]:

$$
S_{k}^{!} / D^{k-1}\left(M_{2-k}^{!}\right) \cong S_{k} \oplus S_{k},
$$


from which (9) follows easily by a dimension argument. This suggests that the "multiplicity two" Hecke theory in [1] can be extended to include the Eisenstein series $G_{k}$ and a one-dimensional subspace of $S_{k}^{!}$whose nonzero forms have the same Hecke eigenvalues as $G_{k}$. This motivates us to refine the notion of Hecke eigenform.

Let $m \geq 2$ be an integer, and let $T(m)$ be the usual $m$ th weight $k$ Hecke operator. We call $f \in M_{k}^{!}$a Hecke eigenform if for each $T(m)$ there is a unique complex number $\lambda_{m}$ such that

$$
\left(\left.f\right|_{k} T(m)-\lambda_{m} f\right)(z) \in D^{k-1}\left(S_{2-k}^{!}\right) .
$$

Two remarks.

1) Our definition includes classical (holomorphic) Hecke eigenforms and Hecke eigenforms with respect to $S_{k}^{!} / D^{k-1}\left(M_{2-k}^{!}\right)$as defined in [1]. Also, it is easily seen that the space $D^{k-1}\left(S_{2-k}^{!}\right)$does not contain any Hecke eigenforms because for every $m$, the choice of $\lambda_{m}$ will not be unique.

2) Manin defined an action of Hecke operators on period polynomials in [ 6 ] which is compatible with the Hecke action on the associated modular forms. This action can be extended to period functions by using the more general definition of Hecke eigenform given here.

A routine calculation now gives us our next result, from which the extension of the "multiplicity two" theorem (Theorem 1.5 in [1]) immediately follows.

Proposition. Let $F \in M_{2-k}^{!}$with nonzero constant term. Then $f=D^{k-1}(F) \in S_{k}^{!}$ is a Hecke eigenform with the same eigenvalues as the Eisenstein series $G_{k}$.

Two remarks.

1) Forms satisfying our hypothesis always exist by Proposition 2.3 of $[1$.

2) Choose $F \in M_{2-k}^{!}$with constant term equal to $\zeta(k-1)$. If $f_{k}=D^{k-1}(F)-G_{k}$, then a simple calculation reveals that the eigenforms $f_{k}$ and $G_{k}$ are orthogonal with respect to the extended Petersson inner product (introduced below).

To state our last result, we introduce Petersson's inner product which is the Hecke equivariant (i.e. $\left.\left(f, g \mid{ }_{k} T(m)\right)=\left(\left.f\right|_{k} T(m), g\right)\right)$ and Hermitian (i.e. $(f, g)=$ $\overline{(g, f)})$ scalar product defined by

$$
(f, g):=\int_{\mathbb{H} / \Gamma} f(\tau) \overline{g(\tau)} y^{k-2} d x d y
$$

for cusp forms $f$ and $g$. Haberland's identity expresses Petersson's inner product as a bilinear form in periods and was derived by Kohnen and Zagier in [5. In [1, the identity was formally extended to the space $M_{k}^{!}$, and here, using the normalized periods in (6), we are able to express Haberland's identity in a much more concise and beautiful way.

Theorem 3. Let $f, g \in M_{k}^{!}$. Then the extended Petersson inner product (as in [1]) may be expressed as

$$
(f, g)=\frac{1}{3(2 i)^{k-1}} \sum_{\substack{m<n \\ m \neq n}} \delta(n) \delta(m) \frac{\Gamma(n+1) \Gamma(k-1-m)}{\Gamma(n+1-m) \Gamma(k-1)} \widehat{r}_{n}(f) \overline{\widehat{r}_{m}(g)},
$$

where $\delta(x):=1+\delta_{-1, x}+\delta_{k-1, x}$ and $\delta_{y, x}$ is Kronecker's delta function. 
Remark 4 . The $\Gamma$ quotient above suggests that certain summands may be undefined when there are poles. To interpret the above formula correctly, we must consider the $\Gamma$ quotient together with the corresponding normalized periods. In this way, the number of zeros will always outnumber the amount of poles that appear, and so we interpret such summands as vanishing.

Proof. In identity (1.5) of 1, periods were defined slightly differently than we did here in identities (44) and (7). Therefore, we write the relation among the various types of periods as

$$
\widehat{r}_{n}(f)=\left(\begin{array}{c}
k-2 \\
n
\end{array}\right) r_{n}(f)=i^{n+1}\left(\begin{array}{c}
k-2 \\
n
\end{array}\right) \dot{r}_{n}(f),
$$

where $0 \leq n \leq k-2$ and $\dot{r}_{n}(f):=\int_{0}^{\infty} f(i t) t^{n} d t$ (i.e. periods of $f$ as defined in [1]).

Haberland's identity in (4.3) of [1] is written as

$$
\begin{aligned}
3 \cdot 2^{k-1}(f, g)= & \sum_{\substack{0 \leq m<n \leq k-2 \\
m \neq \equiv n}} i^{n+1-m}\left(\begin{array}{c}
k-2 \\
n
\end{array}\right)\left(\begin{array}{c}
n \\
m
\end{array}\right) \dot{r}_{n}(f) \overline{\dot{r}_{k-2-m}(g)} \\
& +2 \sum_{\substack{0 \leq n \leq k-2 \\
n \equiv 0}} i^{k-n}\left(\begin{array}{c}
k-1 \\
n+1
\end{array}\right)\left(\overline{\dot{r}_{n}(g)} \frac{a_{f}(0)}{k-1}+\dot{r}_{n}(f) \frac{\overline{a_{g}(0)}}{k-1}\right) .
\end{aligned}
$$

Making use of (10), we rewrite the above identity as

$$
\begin{aligned}
3 \cdot 2^{k-1}(f, g)= & \sum_{\substack{0 \leq m<n \leq k-2 \\
m \neq n}} \delta(m) \delta(n)\left(\begin{array}{c}
n \\
m
\end{array}\right)\left(\begin{array}{c}
k-2 \\
m
\end{array}\right)^{-1} i^{1-k} \widehat{r}_{n}(f) \overline{\widehat{r}_{m}(g)} \\
& +\sum_{\substack{0 \leq m \leq k-2 \\
m \neq k-1}(\bmod 2)} \delta(m) \delta(k-1) \frac{k-1}{m+1} i^{1-k} \widehat{r}_{k-1}(f) \overline{\widehat{r}_{m}(g)} \\
& +\sum_{\substack{0 \leq n \leq k-2 \\
n \neq-1}} \delta(-1) \delta(n) \frac{k-1}{n+1} i^{1-k} \widehat{r}_{n}(f) \overline{\widehat{r}}_{-1}(g) . \\
&
\end{aligned}
$$

Now apply the substitution $m \rightarrow k-2-m$ to the second sum above. Then use the period relations, $\widehat{r}_{k-2-m}(g)=\widehat{r}_{m}(g)$, and rewrite the binomial coefficients and fractions in terms of the $\Gamma$ function to obtain

$$
3(2 i)^{k-1}(f, g)=\sum_{\substack{-1 \leq m<n \leq k-1 \\ m \neq n}} \delta(n) \delta(m) \frac{\Gamma(n+1) \Gamma(k-1-m)}{\Gamma(n+1-m) \Gamma(k-1)} \widehat{r}_{n}(f) \overline{\widehat{r}_{m}(g)} .
$$

Extending the summation over all integers $m$ and $n$ gives the result.

\section{REFERENCES}

1. K. Bringmann, P. Guerzhoy, Z. Kent, and K. Ono, Eichler-Shimura theory for mock modular forms, preprint.

2. J. Bruinier, K. Ono, and R. Rhoades, Differential operators for harmonic weak Maass forms and the vanishing of Hecke eigenvalues, Math. Ann. 342 (2008), 673-693. MR2430995|(2009f:11046)

3. P. Guerzhoy, Hecke operators for weakly holomorphic modular forms and supersingular congruences, Proc. Amer. Math. Soc. 136 (2008), 3051-3059. MR2407067 (2009e:11089)

4. K. Haberland, Perioden von Modulformen einer Variabler und Gruppencohomologie. I, II, III, Math. Nachr. 112 (1983), 245-282, 283-295, 297-315. MR726861 (85k:11022) 
5. W. Kohnen and D. Zagier, Modular forms with rational periods. Modular forms (Durham, 1983), 197-249, Ellis Horwood Ser. Math. Appl.: Statist. Oper. Res., Horwood, Chichester, 1984. MR803368 (87h:11043)

6. Y. Manin, Periods of parabolic forms and $p$-adic Hecke series, Math. USSR Sbornik 21 (1973), 371-393.

7. D. Zagier, Periods of modular forms and Jacobi theta functions, Invent. Math. 104 (1991), 449-465. MR1106744 (92e:11052)

Department of Mathematics and Computer Science, Emory University, Atlanta, GeOrgia 30322

E-mail address: kent@mathcs.emory.edu 